\title{
On the Difference between Self-Assembling Process of Monomeric and Dimeric Surfactants with the Same Head to Tail Ratio: A Lattice Monte Carlo Simulation
}

\author{
Reza Behjatmanesh-Ardakani and Maryam Farsad \\ Department of Chemistry, Payame Noor University, P.O. Box 19395-3697, Tehran, Iran \\ Correspondence should be addressed to Reza Behjatmanesh-Ardakani; reza_b_m_a@yahoo.com
}

Received 26 June 2012; Revised 8 August 2012; Accepted 9 August 2012

Academic Editor: André Silva Pimentel

Copyright (C) 2013 R. Behjatmanesh-Ardakani and M. Farsad. This is an open access article distributed under the Creative Commons Attribution License, which permits unrestricted use, distribution, and reproduction in any medium, provided the original work is properly cited.

\begin{abstract}
Experimental data show that gemini surfactants have critical micelle concentrations that are almost tenfold lower than the CMCs of single chain ones. It is believed that the spacer groups play an important role in this subject. Short hydrophilic or long hydrophobic spacers can reduce CMC dramatically. In this paper, self-assembling processes of double-chain and one-chain surfactants with the same head to tail ratio are compared. Dimeric chain structure is exactly double of single chain. In other words, hydrophiliclyophilic balances of two chain models are the same. Two single chains are connected head-to-head to form a dimeric chain, without introducing extra head or tail beads as a spacer group. Premicellar, micellar, and shape/phase transition ranges of both models are investigated. To do this, lattice Monte Carlo simulation in canonical ensemble has been used. Results show that without introducing extra beads as spacer group, the CMC of $\left(\mathrm{H}_{3} \mathrm{~T}_{3}\right)_{2}$ as a dimeric surfactant is much lower than the CMC of its similar single chain, $\mathrm{H}_{3} \mathrm{~T}_{3}$. For dimeric case of study, it is shown that bolaform aggregates are formed.
\end{abstract}

\section{Introduction}

Gemini surfactants are relatively new type of surface active materials. They can self-assemble in a wide range from nanoscale (spherical micelles) to meso-scale aggregates (vesicles, monolayers, bilayers, and so on) [1]. A gemini surfactant contains two conventional single chain surfactant whose heads are connected by a hydrophobic or hydrophilic part named as spacer [2]. Gemini surfactants have very low critical micelle concentration (CMC) compared with the corresponding single chain surfactants. Unlike to the conventional amphiphile molecules with spherical micelles, gemini surfactants form unusual aggregation morphologies such as vesicles and bilayers [3, 4].

There are many experimental studies on gemini surfactants; however, surprisingly, only a few theoretical studies are found in the literature. The first molecular dynamics simulation of gemini amphiphiles has been conducted by Karaborni et al. [5], who used coarse-grained model to simulate gemini surfactants with different hydrophobic spacer lengths. Their results showed that the structures of aggregates depend on the number of oil-like spacer between head parts. With single spacer, linear thread-like micelles are formed and for longer spacer, mixture of spheroid and tree-like aggregates are produced. Using Smit's model [6], Maiti et al. developed a simple coarse-grained model of surfactant oligomers containing dimeric and trimeric amphiphiles [7]. Their molecular dynamics simulation results exhibited formation of closed-loop micelles for dimeric and trimeric surfactants. Wu et al. performed large scale coarse-grained molecular dynamics simulations to study self-assembling process of $12-S-12$ gemini surfactants with $S=6,12$, and 20 [8]. In their study, free gemini molecules aggregate into the oligomers (premicelles) and then these small clusters merge with each other to form larger clusters such as vesicles.

The first Monte Carlo simulation study on the selfassembling process of lattice gemini amphiphiles goes back to the work of Bernardes [9]. In later work, the author has tested effects of chain rigidity on the aggregate shapes [10]. Maiti and Chowdhury carried out Monte Carlo simulation 
similar to the Bernardes' model and investigated three factors containing length of spacer, length of tail, and bending rigidity of spacer and/or tails on the critical micelle concentration of a lattice gemini amphiphile [11, 12]. They have shown, in agreement to the experimental results, their simulated CMC reached a maximum at a certain length of spacer. In later work, Maiti and his coworkers studied cross-linking between one-tailed micelles by gemini surfactants [13]. Layn et al. have used lattice Monte Carlo and quasichemical theory to investigate phase behavior of simple gemini surfactant with only hydrophilic spacer [14]. They have studied effects of oil length, surfactant rigidity, and temperature on the phase behavior. Recently, Davis and Panagiotopoulos have used grand canonical Monte Carlo simulation of mixture of one-tailed and double-tailed nonionic amphiphile to study the effect of addition of a micellizing amphiphile on the behavior of another phase separating amphiphile [15]. Very recently, Jackson et al. investigated assembling processes of two-headed linear surfactants by two dimensional lattice Monte Carlo simulations [16].

In the present work, we have compared self-assembling process for two lattice amphiphiles with the same head to tail ratio. Both of amphiphiles are symmetrical. Dimeric chains are double of single chains that connected head-to-head. In this work, we have not considered any extra hydrophilic or hydrophobic beads between heads as a spacer group to investigate how aggregates are formed without spacer groups. Meanwhile, different thermodynamic behaviors are compared including $\mathrm{CMC}$, premicellar concentration, size distribution, polydispersity, and aggregation number. It is shown that transition points in graphs of polydispersity, aggregation number, and premicellar concentration, match with the CMC point in graph of monomer concentration.

\section{Model}

The model is based on the hydrophobic interactions as a main driving force of micellization process. Details of the model can be found in previous studies [17, 18]. Here, we only summarize it. A simple cubic lattice with the size of $80 \times 80 \times 80,100 \times 100 \times 100$, or $200 \times 200 \times$ 200 is used as a box of simulation. In all simulations, a water molecule occupies only a single lattice site. Surfactant molecules are modeled as short chains on the lattice. Onetailed and double-tailed surfactants are modeled by $\mathrm{H}_{i} \mathrm{~T}_{j}$ and $\left(\mathrm{H}_{i} \mathrm{~T}_{j}\right)_{2}$ or $\mathrm{T}_{j} \mathrm{H}_{2 i} \mathrm{~T}_{j}$, respectively. " $\mathrm{H}$ " and " $\mathrm{T}$ " denote a head and a tail, respectively. The integers " $i$ " and " $j$ " denote the length of head and tail parts, respectively. In this study, symmetrical monomeric and dimeric surfactants with $i=$ $j=3$ are considered. The structures of a monomeric and a dimeric lattice surfactant have been shown in Figure 1. A lattice bead interacts only with its six nearest neighbor beads, thus the coordination number is equal to six $(z=$ 6). Periodic boundary conditions and excluded volumes are used to mimic the box of simulation as the bulk of solution. Canonical ensemble with the constants of $N, V$, and $\mathrm{T}$ is used

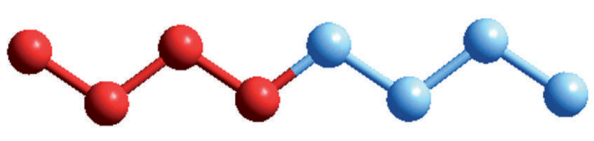

(a)

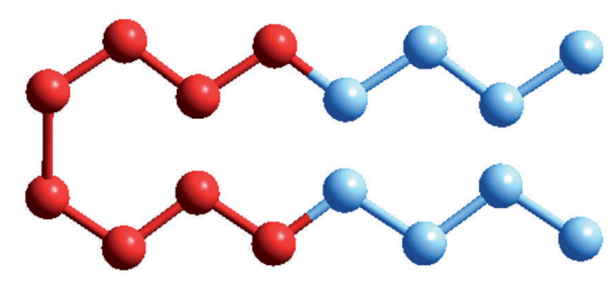

(b)

FIGURE 1: Structure of (a) monomeric and (b) dimeric studied lattice surfactants. Red beads are heads and light blue beads are tails.

for ensemble-averaging. The potential model is as previous and defined as follows $[17,18]$ :

$$
\frac{E}{k_{B} \mathrm{~T}}=\frac{\varepsilon}{k_{B} \mathrm{~T}}\left(n_{\mathrm{T}, W}+n_{\mathrm{T}, \mathrm{H}}\right),
$$

where $n_{\mathrm{T}, W}$ and $n_{\mathrm{T}, \mathrm{H}}$ are the numbers of tail-water and tailhead pairs, respectively, and $\varepsilon /\left(k_{B} \mathrm{~T}\right)$ is the dimensionless interactions parameter. In the present work, we have set $\varepsilon /\left(k_{B} \mathrm{~T}\right)=0.7$. Previously, it has been shown that this value of interaction parameter gives rise to micellization [18]. Only hydrophobicity of surfactant molecules, as a main characteristic of amphiphile molecules, is considered in the potential model.

We have used reptation [19] and configurational bias Monte Carlo [20] algorithms to move surfactant molecules. Our simulations are based on the standard Metropolis algorithm in which the probability of $P=\operatorname{Min}(1, \exp (-\beta \Delta E))$ for reptation and $P=\operatorname{Min}\left(1,(W(n)) /(W(o)) e^{-\beta \Delta E}\right)$ for configurational bias Monte Carlo moves are used, where $\Delta E$ is the difference of the total energy between new trial and old configurations, $\beta$ is $1 /\left(k_{B} \mathrm{~T}\right)$, and $P$ is the probability by which new trial configuration is accepted. The Rosenbluth weight $(W)$ is number of solvent sites $\left(z_{s}(i)\right)$ around the next bead to be removed or regrown.

Source codes were written in Fortran77 language, and compiled with intel Fortran Composer Xe compiler under the Centos linux. Nearly $10^{11}$ moves have been used to equilibrate initial configurations, and $2 \times 10^{11}$ moves were used for ensemble averaging. 2000 snapshots have been used for ensemble averaging to get smooth graphs.

2.1. Definition of Parameters. Two kinds of concentration have been defined: total volume fraction $\left(V_{\text {tot }}\right)$ and cluster number density $\left(X_{n}\right)$ with aggregation number $n(n \geq 1)$,

$$
\begin{gathered}
V_{\text {tot }}=\frac{\text { total sites of surfactant molecules }}{\text { total sites of lattice }} \\
X_{n}=\frac{\text { total number of molecules in cluster }}{\text { total sites of the lattice }} .
\end{gathered}
$$


According to $X_{n}$, concentration of free surfactant molecules is defined as:

$$
X_{1}=\frac{\text { total number of free molecules }}{\text { total sites of the lattice }} .
$$

A surfactant is considered as a free molecule whenever all nearest neighbors of its tail sites are water molecules. Two surfactant molecules are considered to be in the same cluster if at least one tail site of the first molecule is the nearest neighbor of any tail site of the second molecule. Premicelles are small aggregates, usually with aggregation number less than ten [21]. Premicellar concentration is defined as $\sum_{n=2}^{10} n X_{n}$. Polydispersity is defined as $I_{P}=$ $N_{w} / N_{n}$, where $N_{n}$ is number-averaged aggregation number, and $N_{w}$ is weight-averaged aggregation number. $N_{n}$ and $N_{w}$ are defined as follows:

$$
\begin{gathered}
N_{n}=\frac{\sum_{n=2}^{n_{\max }} n X_{n}}{\sum_{n=2}^{n_{\max }} X_{n}}, \\
N_{w}=\frac{\sum_{n=2}^{n_{\max }} n^{2} X_{n}}{\sum_{n=2}^{n_{\max }} n X_{n}} .
\end{gathered}
$$

A free molecule is not considered in the above summation, and $n_{\max }$ is the maximum aggregation number in all of the snapshots considered in the ensemble averaging.

\section{Results and Discussion}

Surfactant solutions may be classified into three concentration ranges: premicellar, micellar, and phase/shape transition ranges. For dimeric surfactants, almost all previous studies focused on the micellar and/or phase/shape transition ranges. In this paper, properties of single and double chain surfactant solution in three above ranges are reviewed and compared.

Premicelles are aggregates with small aggregation number. Often, clusters with aggregation number less or equal to ten are referred as premicelles [21]. Figures 2 and 3 show the premicellar concentration of single and double chain surfactants. The graphs show that the concentration of premicelles for single chain surfactant is higher than nearly tenfold of that of dimeric one. At first, premicellar concentration increases in sigmoidal fashion up to a maximal value, and then relaxes to the lower values. This relaxation is much slower for dimeric chain compared to monomeric surfactant. This behavior is very similar to the graphs of CMC determination (Figures 4 and 5).

The second range belongs to the micellar phase. The concentration at which micelles begin to be formed is named as critical micelle concentration (CMC). Various definitions for the CMC have been used in the literature. We have defined CMC as a surfactant concentration at which free molecules reach maximal value. Figures 4 and 5 show CMC graphs for both single and double chain surfactants. On the basis of the definition, $\mathrm{CMC}$ for $\mathrm{H}_{3} \mathrm{~T}_{3}$ is nearly equal to $V_{\text {tot }}=0.06$, and for $\left(\mathrm{H}_{3} \mathrm{~T}_{3}\right)_{2}$ is equal to $V_{\text {tot }}=0.014$. $\mathrm{CMC}$ of dimeric surfactant is much lower than monomeric one. As is clear from above figures, premicellar behaviors are similar to the free surfactant concentration changes.

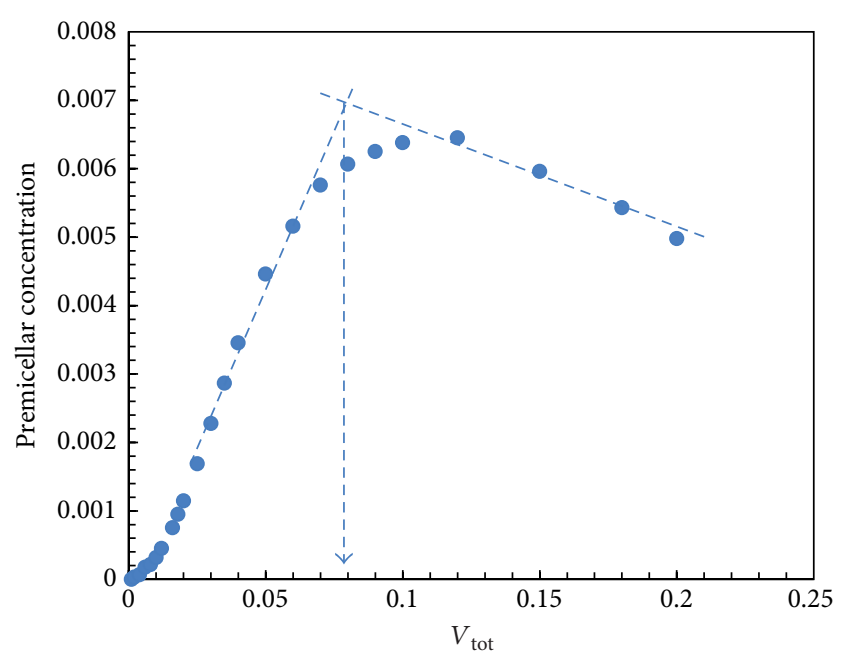

FIgURE 2: Premicellar number density (summation of $n X_{n}$ with $2 \leq n \leq 10)$ versus total volume fraction $\left(V_{\text {tot }}\right)$ of surfactant. The graph belongs to $\mathrm{H}_{3} \mathrm{~T}_{3}$ as a single chain surfactant. Maximum concentration is found near to CMC. After CMC, premicelles reduce rapidly.

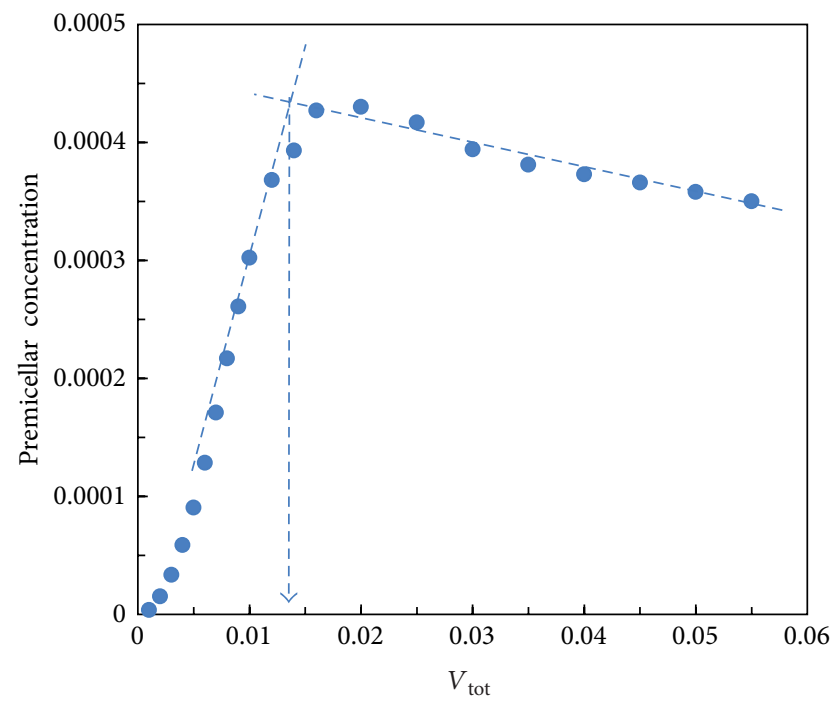

Figure 3: Premicellar number density (summation of $n X_{n}$ with $2 \leq n \leq 10)$ versus total volume fraction $\left(V_{\text {tot }}\right)$ of surfactant. The graph belongs to $\left(\mathrm{H}_{3} \mathrm{~T}_{3}\right)_{2}$ as a double chain surfactant. Maximum concentration is found near to CMC. After CMC, premicelles remain nearly constant.

At CMC, premicelles reach maximal values, and at higher concentration small aggregates grow and form larger clusters. After CMC, premicellar concentration reduces and system tends to be more monodispersed. Polydispersity is defined as the ratio of weight-averaged aggregation number $\left(N_{w}\right)$ to number-averaged aggregation number $\left(N_{n}\right)$. Figures 6 and 7 show polydispersity changes for single and double chain surfactants. Data show that for both systems there is a break point on the curve of polydispersity. This occurs at 


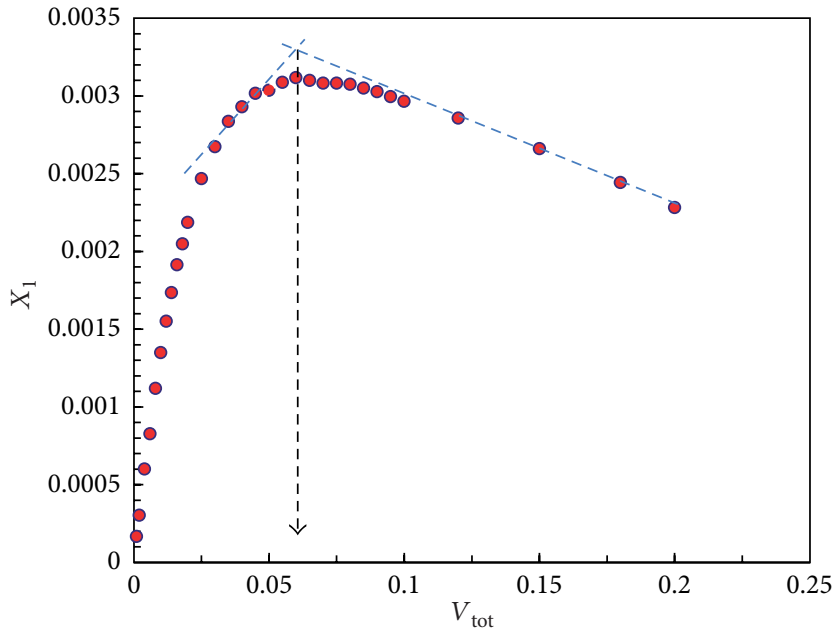

FIGURE 4: Free surfactant molecule number density $\left(X_{1}\right)$ versus total volume fraction $\left(V_{\text {tot }}\right)$. The graph belongs to $\mathrm{H}_{3} \mathrm{~T}_{3}$ as a single chain surfactant. This graph is often used for determination of the CMC.

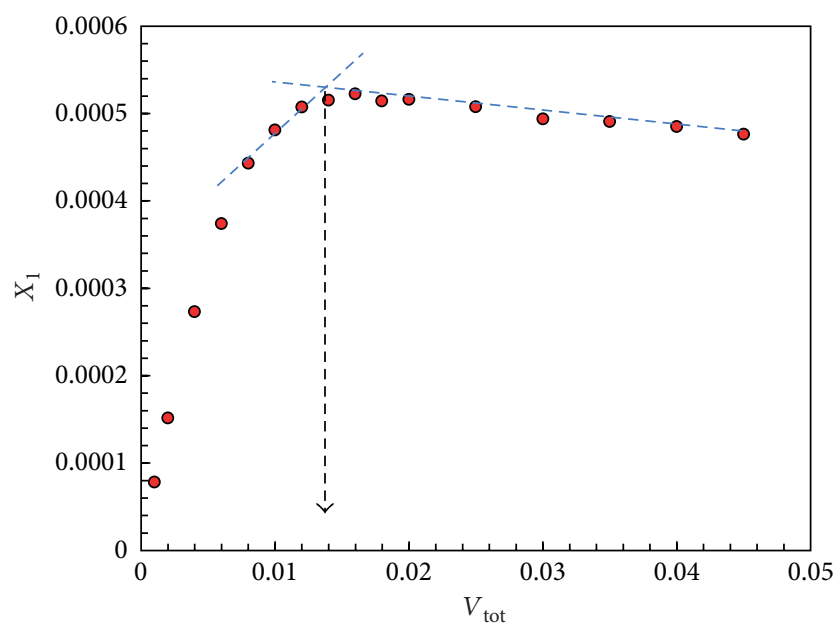

FIGURE 5: Free surfactant molecule number density $\left(X_{1}\right)$ versus total volume fraction $\left(V_{\text {tot }}\right)$. The graph belongs to $\left(\mathrm{H}_{3} \mathrm{~T}_{3}\right)_{2}$ as a double chain surfactant. This graph is often used for determination of the CMC.

volume fractions of 0.09 and 0.018 for $\mathrm{H}_{3} \mathrm{~T}_{3}$ and $\left(\mathrm{H}_{3} \mathrm{~T}_{3}\right)_{2}$, respectively.

Another important issue in the micellar phase is about cluster size distribution. It is believed that CMC relates to the horizontal inflection point at cluster size distribution [22]. Figures 8 and 9 show the inflection point at cluster size distribution for single and double chain surfactants, respectively. The inflection point occurs at concentration of 0.1 and 0.015 for single and double chain surfactant, respectively. Comparing these values with the critical points of Figures 2 to 7 (which can be named as their CMCs), it shows that there is a close connection between inflection points in above figures (Figures 8 and 9) and critical points of Figure 2 to Figure 7, that is, the inflection points can be

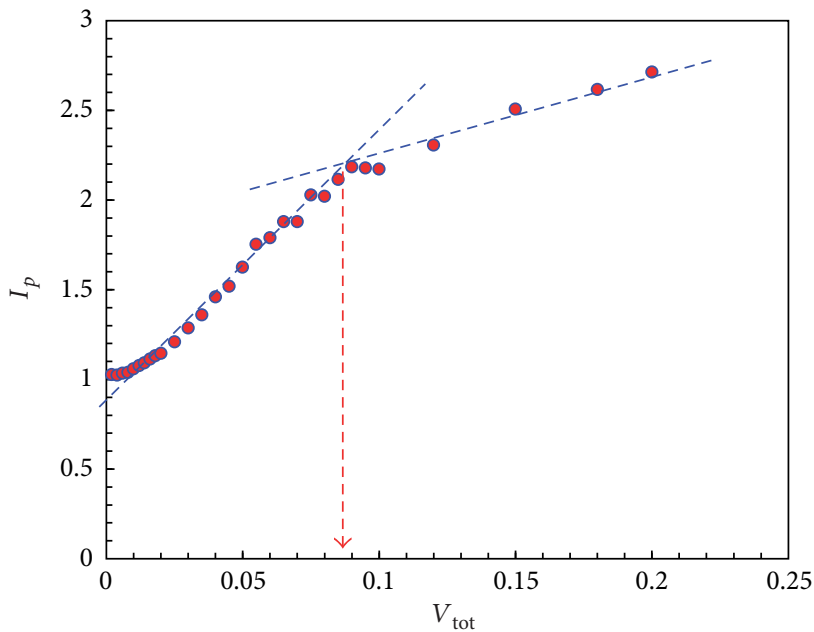

FIGURE 6: Polydispersity $\left(I_{P}\right)$ versus total volume fraction $\left(V_{\text {tot }}\right)$ for $\mathrm{H}_{3} \mathrm{~T}_{3}$ as a single chain surfactant. From this graph CMC is estimated by 0.09 . The slope of the graph reduces after CMC.

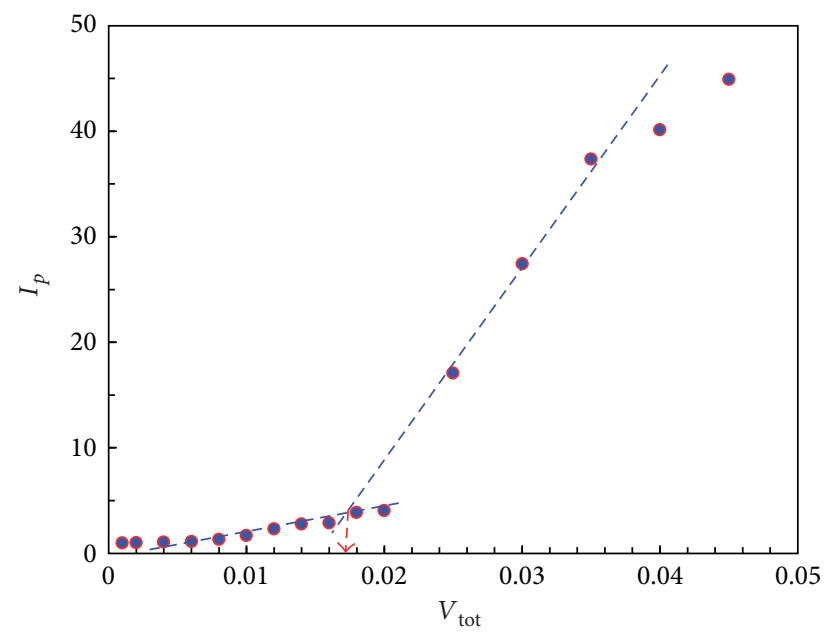

FIgURe 7: Polydispersity $\left(I_{P}\right)$ versus total volume fraction $\left(V_{\text {tot }}\right)$ for $\left(\mathrm{H}_{3} \mathrm{~T}_{3}\right)_{2}$ as a double chain surfactant. From this graph CMC is estimated nearly 0.02 . The slope of the graph increases after CMC.

considered as CMC points. Similarity of these critical values shows that the idea of [22] is correct.

The third concentration range of surfactant systems contains shape/phase transition. In some papers, additional peaks in cluster size distribution have been interpreted as a clue for shape/phase transition in surfactant systems [23]. In our previous paper, we have proved that a true simulation should have one peak in cluster size distribution [18]. Figure 10 shows the cluster size distribution for double chain surfactant. It shows that the peak in the distribution shifts to higher values at higher concentration. We have repeated simulation for greater box $100 \times 100 \times 100$ to test the size effect. Figure 11 shows the results for greater box. There are two important points on this figure. First, as for smaller box, peak shifts to larger values, and second, for greater lattice at 


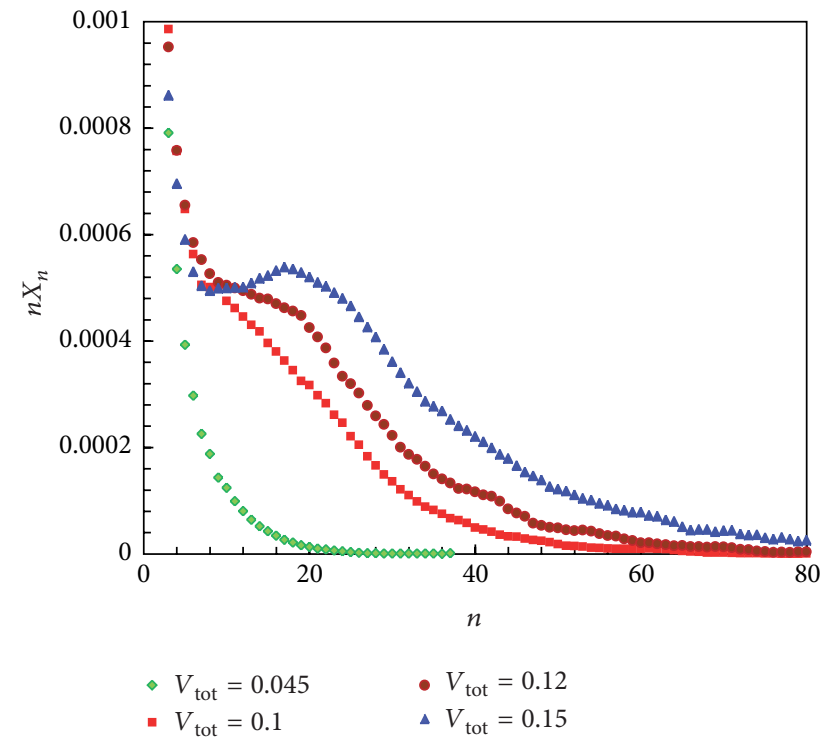

FIGURE 8: Cluster size distribution $\left(n X_{n}\right)$ for the micellar range of concentration of $\mathrm{H}_{3} \mathrm{~T}_{3}$ as a single chain surfactant. Inflection point occurs at $V_{\text {tot }}=0.1$ which agree with $\mathrm{CMC}$ of $\mathrm{H}_{3} \mathrm{~T}_{3}$.

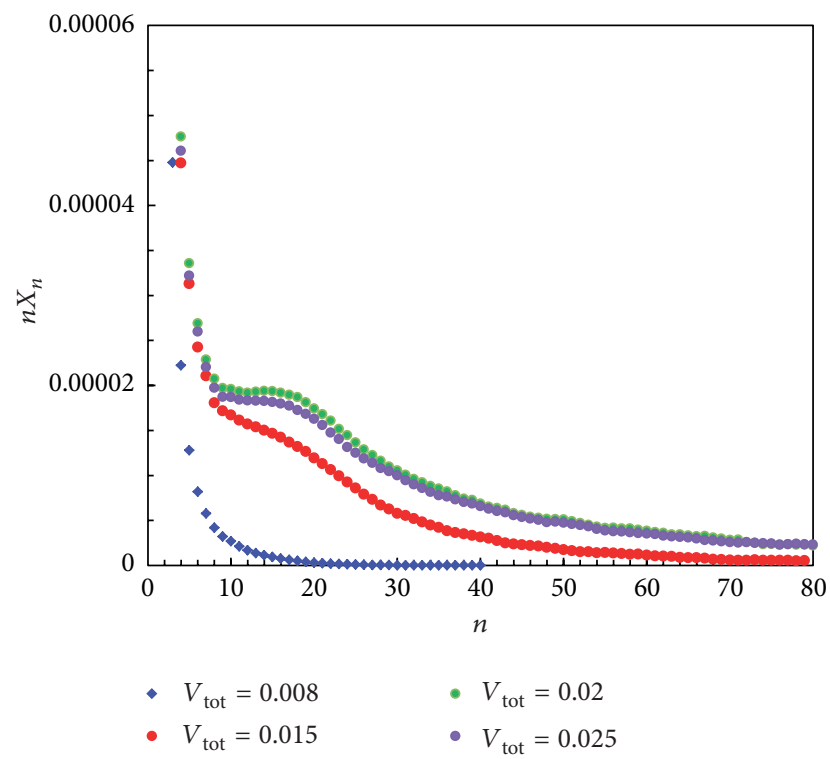

Figure 9: Cluster size distribution $\left(n X_{n}\right)$ for the micellar range of concentration of $\left(\mathrm{H}_{3} \mathrm{~T}_{3}\right)_{2}$ as a double chain surfactant. Inflection point occurs at $V_{\text {tot }}=0.016$ which agree with $\mathrm{CMC} \mathrm{of}\left(\mathrm{H}_{3} \mathrm{~T}_{3}\right)_{2}$.

a certain concentration, peak appeared at higher aggregation number. It can be concluded that for double chain surfactant, with enlarging the box to infinitive, aggregation number becomes very large, that is, phase separation occurs. On the other hand, for $\mathrm{H}_{3} \mathrm{~T}_{3}$, even at high concentration of 0.15 , there is not any shift in the position of peak. Another clue for this subject is tracing weight-average aggregation number $\left(N_{w}\right)$. Figures 12 and 13 show $N_{w}$ for single and double chain surfactants. As is clear for double chain surfactant, $N_{w}$ increases rapidly.

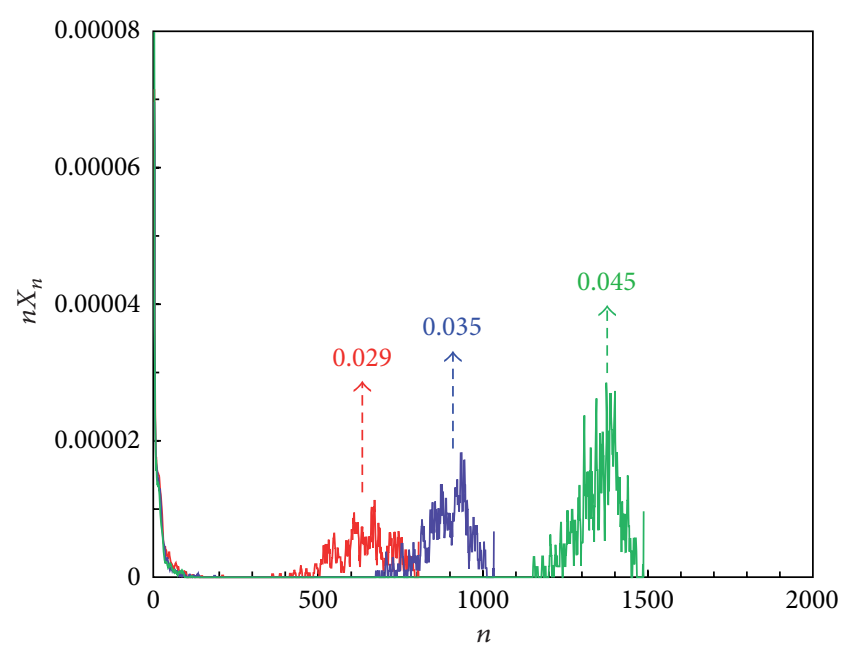

Figure 10: Cluster size distribution $\left(n X_{n}\right)$ for the phase transition range of concentration of $\left(\mathrm{H}_{3} \mathrm{~T}_{3}\right)_{2}$ as a double chain surfactant. Peak of distribution shifts to higher values of aggregation number. Lattice size is $80 \times 80 \times 80$.

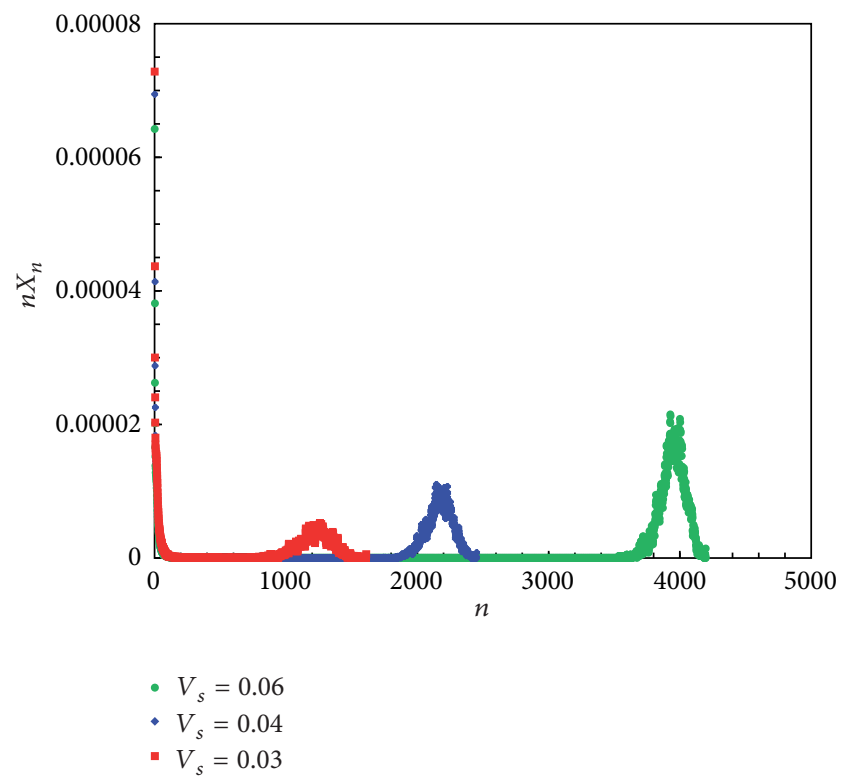

Figure 11: Same as Figure 9 but for $100 \times 100 \times 100$ lattice size.

The ratio of head to tail for both single and double chain surfactants is the same. Each dimeric molecule is equal to two single chain molecules, so, it is expected that the properties of single chain molecule to be similar to double chain at twice concentration of dimeric molecule; however, this is not the case. Looking at the structure of aggregates of double chain molecule help us to understand the differences. Figure 14 shows the structures of aggregates at concentrations of $0.016,0.02$, and 0.07 . The figure shows how head groups connect separate micelles. At high concentration we encounter with large bolaform aggregates in which spacer groups play a connection role. Figure 15 shows snapshot of box of simulation at volume fraction of 0.06 . It shows that 


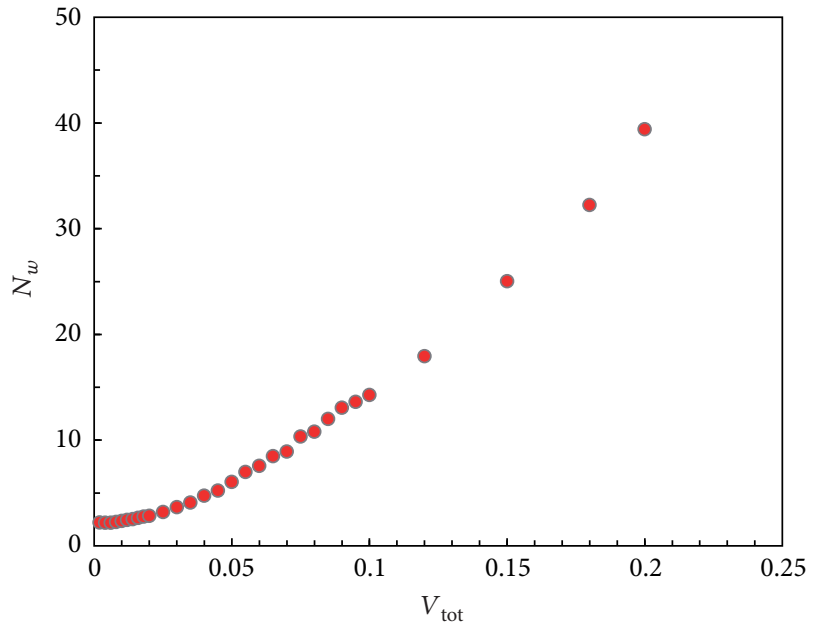

FIgURE 12: Weight-average aggregation number $\left(N_{w}\right)$ for $\mathrm{H}_{3} \mathrm{~T}_{3}$ as a single chain surfactant. Aggregation number increases slowly.

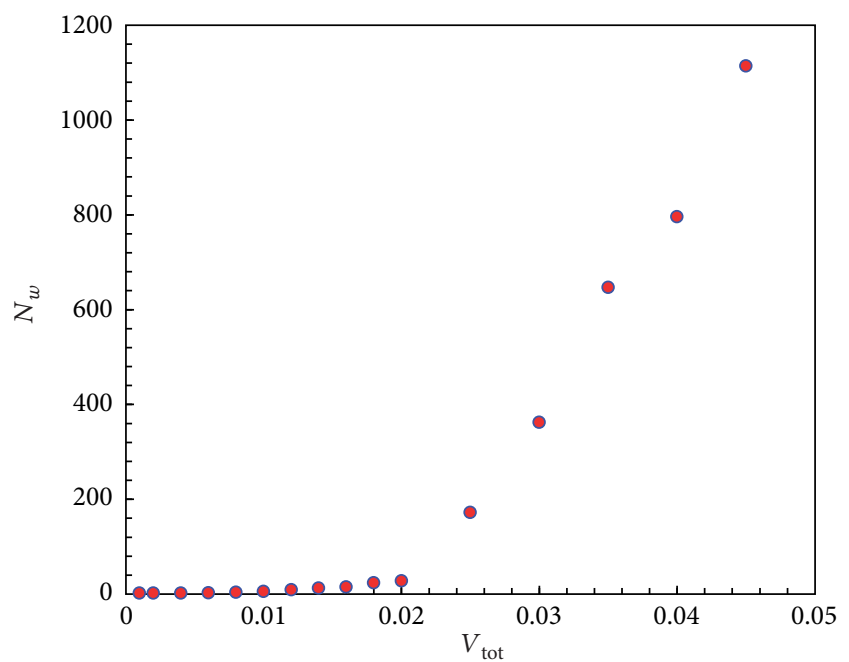

FIGURE 13: Weight-average aggregation number $\left(\mathrm{N}_{w}\right)$ for $\left(\mathrm{H}_{3} \mathrm{~T}_{3}\right)_{2}$ as a double chain surfactant. Aggregation number increases very rapidly.

very large aggregates are formed at nearly low concentration. All results show that to reduce dramatically CMC or increase aggregation number, it is sufficient that two single chains are connected from head positions, and it is not necessary to introduce any extra hydrophobic or hydrophilic spacer groups.

\section{Conclusion}

In this paper, we have chosen very similar single and double chain surfactant with the same head to tail ratio. Each double chain molecule equals to two single chains in number of heads and tails. To build a dimeric chain from two single chains, heads are connected without introducing any hydrophobic or hydrophilic spacer groups. It is shown that

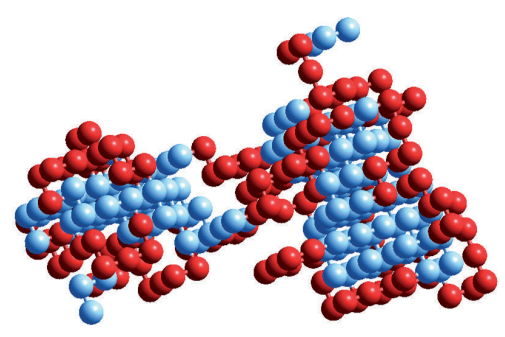

(a) 0.016

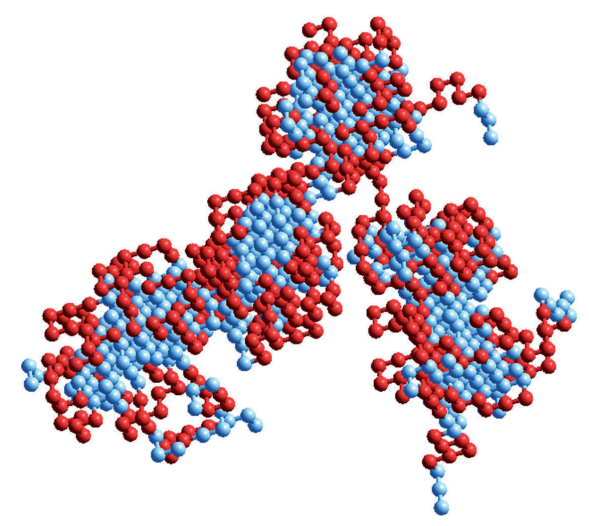

(b) 0.02

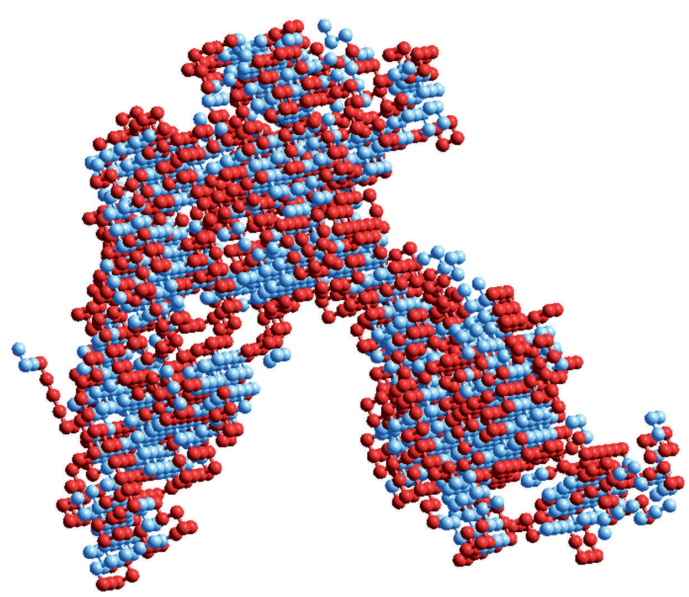

(c) 0.07

FIGURE 14: Structure of aggregates of $\left(\mathrm{H}_{3} \mathrm{~T}_{3}\right)_{2}$ at volume fractions of (a) 0.016 , (b) 0.02 , and (c) 0.07 . The structures show that head groups of the molecule (green beads) play a connector role to join separate micelles. At high concentrations bolaform micelles are formed.

the properties of double chain surfactant are very different from single chain surfactant. Its CMC is nearly tenfold lower than the CMC of single chain molecule; its aggregation number is greater than 30 times of aggregation number of single chain surfactant, and the cluster size distribution shows a kind of phase transition that is not present for single chains. It has been shown that head groups of double chain surfactants as spacer groups are the main factor for these differences. They play a connector role by which separate micelle are joined to each other. In addition, results show that $\mathrm{CMC}$ can be estimated from free monomer concentration, 


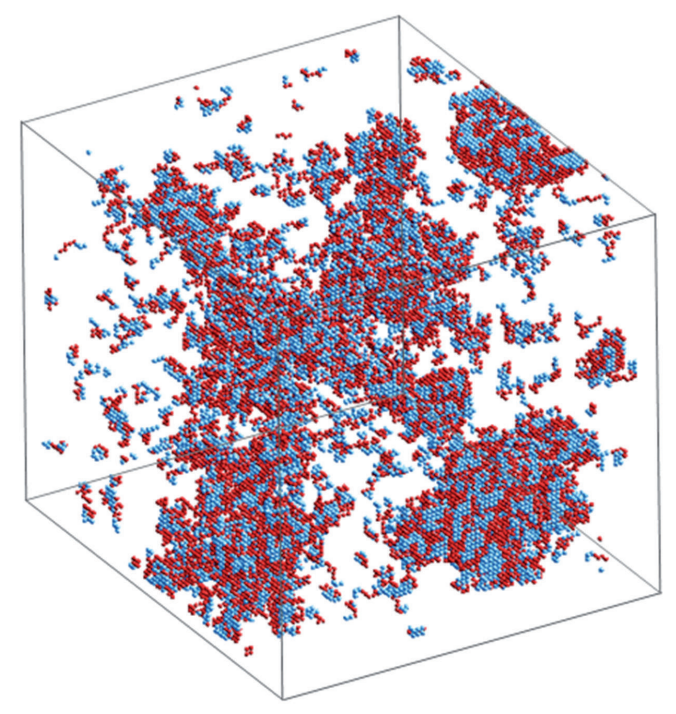

FIGURE 15: Snapshot of box of simulation of $\left(\mathrm{H}_{3} \mathrm{~T}_{3}\right)_{2}$ at volume fraction of 0.06 .

premicellar concentration, aggregation number, and polydispersity graphs. As a main result, it is shown that how without introducing extra hydrophobic or hydrophilic spacer groups, dimeric amphiphiles behave similar to gemini surfactants.

\section{References}

[1] Q. Huo, R. Leon, P. M. Petroff, and G. D. Stucky, "Mesostructure design with gemini surfactants: supercage formation in a threedimensional hexagonal array," Science, vol. 268, no. 5215, pp. 1324-1327, 1995.

[2] R. Zana and J. Xia, Gemini Surfactants: Synthesis, Interfacial and Solution-Phase Behavior, and Applications, Marcel Dekker, New York, NY, USA, 2004.

[3] R. Zana and Y. Talmon, "Dependence of aggregate morphology on structure of dimeric surfactants," Nature, vol. 362, no. 6417, pp. 228-230, 1993.

[4] D. Danino, Y. Talmon, H. Levy, G. Beinert, and R. Zana, "Branched threadlike micelles in an aqueous solution of a trimeric surfactant," Science, vol. 269, no. 5229, pp. 1420-1421, 1995.

[5] S. Karaborni, K. Esselink, P. A. J. Hilbers et al., "Simulating the self-assembly of gemini (dimeric) surfactants," Science, vol. 266, no. 5183, pp. 254-256, 1994.

[6] B. Smit, P. A. J. Hilbers, K. Esselink, L. A. M. Rupert, N. M. Van Os, and A. G. Schlijper, "Computer simulations of a water/oil interface in the presence of micelles," Nature, vol. 348, no. 6302, pp. 624-625, 1990.

[7] P. K. Maiti, Y. Lansac, M. A. Glaser, N. A. Clark, and Y. Rouault, "Self-assembly in surfactant oligomers: a coarsegrained description through molecular dynamics simulations," Langmuir, vol. 18, no. 5, pp. 1908-1918, 2002.

[8] R. Wu, M. Deng, B. Kong, and X. Yang, "Coarse-grained molecular dynamics simulation of ammonium surfactant selfassemblies: micelles and vesicles," Journal of Physical Chemistry $B$, vol. 113, no. 45, pp. 15010-15016, 2009.
[9] A. T. Bernardes, "Monte Carlo simulation of vesicle self-organisation," Journal de Physique II, vol. 6, no. 2, pp. 169-174, 1996.

[10] A. T. Bernardes, "Computer simulations of spontaneous vesicle formation," Langmuir, vol. 12, no. 24, pp. 5763-5767, 1996.

[11] P. K. Maiti and D. Chowdhury, "Micellar aggregates of gemini surfactants: Monte Carlo simulation of a microscopic model," Europhysics Letters, vol. 41, no. 2, pp. 183-188, 1998.

[12] P. K. Maiti and D. Chowdhury, "A microscopic model of gemini surfactants: self-assemblies in water and at air-water interface," Journal of Chemical Physics, vol. 109, no. 12, pp. 5126-5133, 1998.

[13] P. K. Maiti, K. Kremer, O. Flimm, D. Chowdhury, and D. Stauffer, "Cross-linking of micelles by gemini surfactants," Langmuir, vol. 16, no. 8, pp. 3784-3790, 2000.

[14] K. M. Layn, P. G. Debenedetti, and R. K. Prud'homme, "A theoretical study of Gemini surfactant phase behavior," Journal of Chemical Physics, vol. 109, no. 13, pp. 5651-5658, 1998.

[15] J. R. Davis and A. Z. Panagiotopoulos, "Micellization and phase separation in binary amphiphile mixtures," Molecular Physics, vol. 107, no. 22, pp. 2359-2366, 2009.

[16] D. R. Jackson, A. Mohareb, J. MacNeil, M. S. G. Razul, D. G. Marangoni, and P. H. Poole, "Simulations of a lattice model of two-headed linear amphiphiles: influence of amphiphile asymmetry," Journal of Chemical Physics, vol. 134, no. 20, Article ID 204503, 2011.

[17] R. Behjatmanesh-Ardakani, "Polymer-centered theory in comparison with surfactant-centered theory: a lattice Monte Carlo study," Journal of Chemical Physics, vol. 122, no. 20, Article ID 204903, pp. 1-5, 2005.

[18] R. Behjatmanesh-Ardakani, "Additional peaks in the cluster size distribution of amphiphile + water systems: a clue for shape/phase transition or statistical uncertainty," Theoretical Chemistry Accounts, vol. 118, no. 4, pp. 799-805, 2007.

[19] P. G. De Gennes, "Reptation of a polymer chain in the presence of fixed obstacles," The Journal of Chemical Physics, vol. 55, no. 2, pp. 572-579, 1971.

[20] J. I. Siepmann and D. Frenkel, "Configurational bias Monte Carlo: a new sampling scheme for flexible chains," Molecular Physics, vol. 75, pp. 59-70, 1992.

[21] S. K. Talsania, Y. Wang, R. Rajagopalan, and K. K. Mohanty, "Monte Carlo simulations for micellar encapsulation," Journal of Colloid and Interface Science, vol. 190, no. 1, pp. 92-103, 1997.

[22] E. Ruckenstein and R. Nagarajan, "Critical micelle concentration and the transition point for micellar size distribution," Journal of Physical Chemistry, vol. 85, no. 20, pp. 3010-3014, 1981.

[23] M. Lísal, C. K. Hall, K. E. Gubbins, and A. Z. Panagiotopoulos, "Self-assembly of surfactants in a supercritical solvent from lattice Monte Carlo simulations," Journal of Chemical Physics, vol. 116, no. 3, pp. 1171-1184, 2002. 

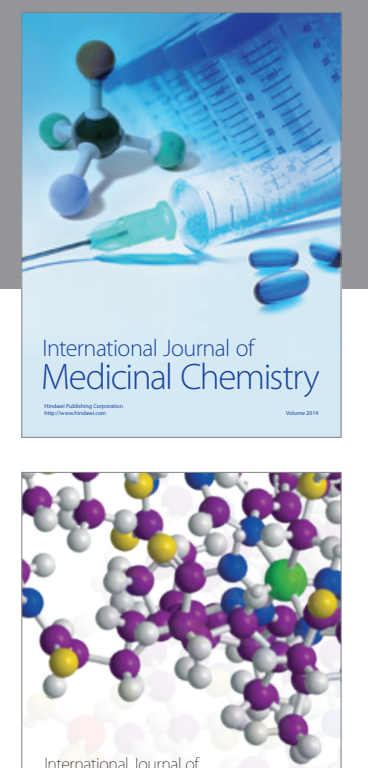

\section{Carbohydrate} Chemistry

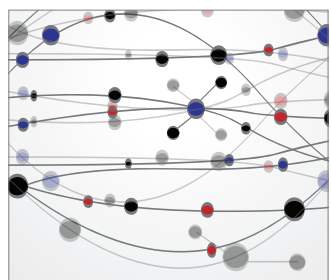

The Scientific World Journal
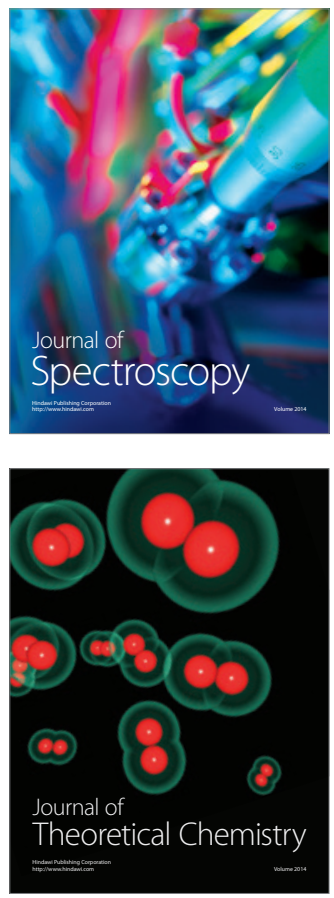
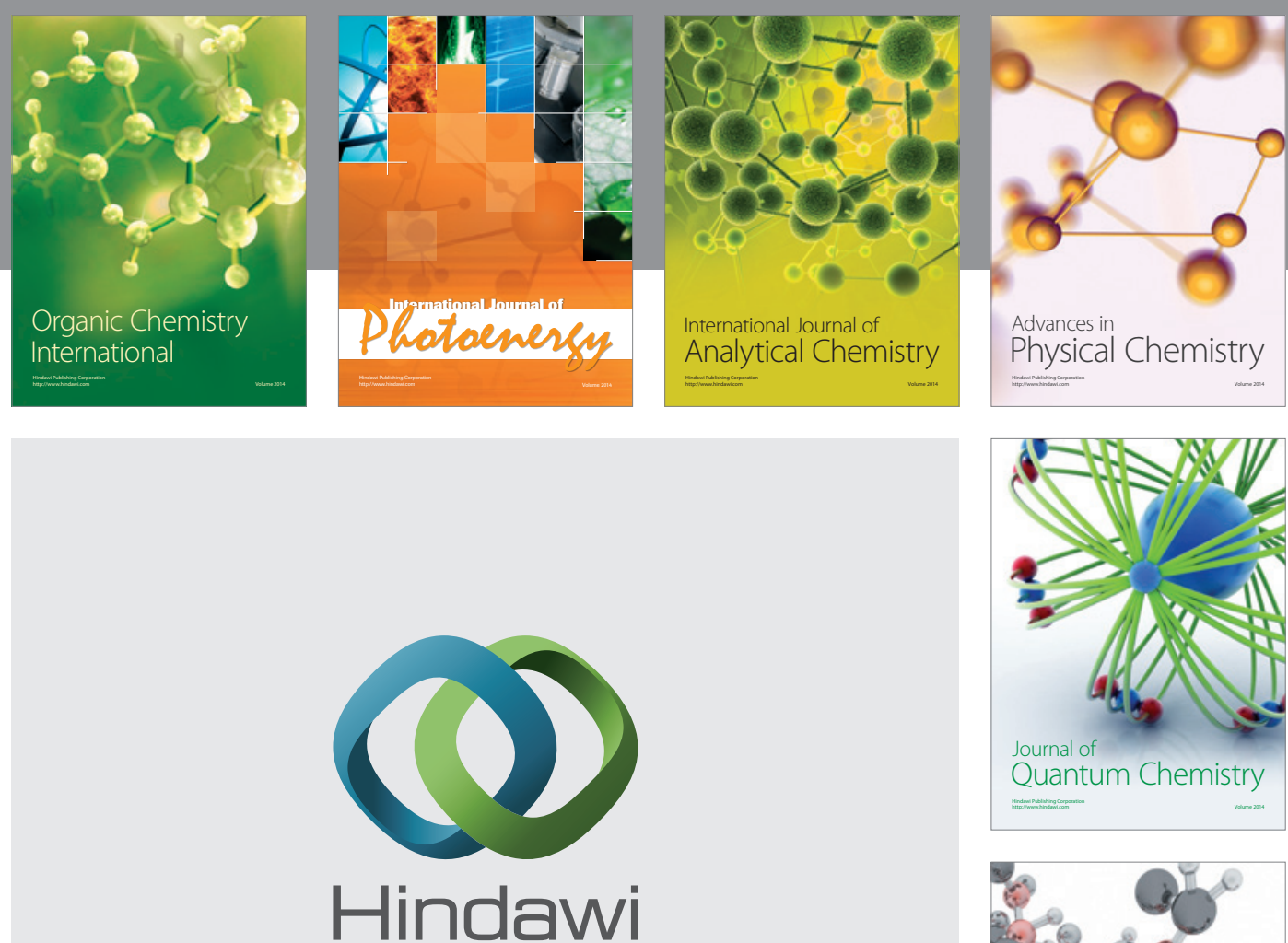

Submit your manuscripts at

http://www.hindawi.com

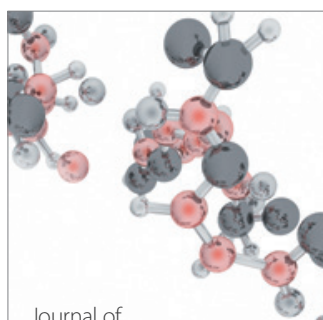

Analytical Methods

in Chemistry

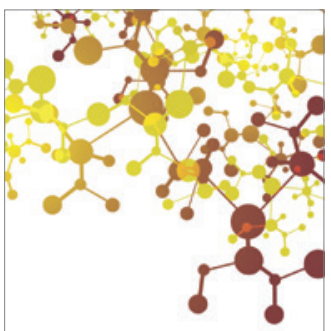

Journal of

Applied Chemistry

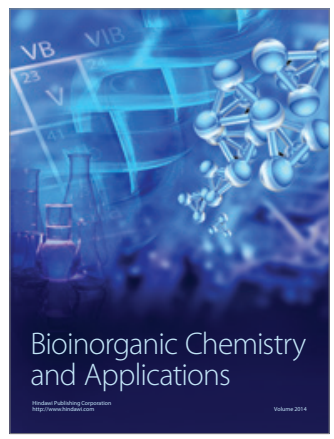

Inorganic Chemistry
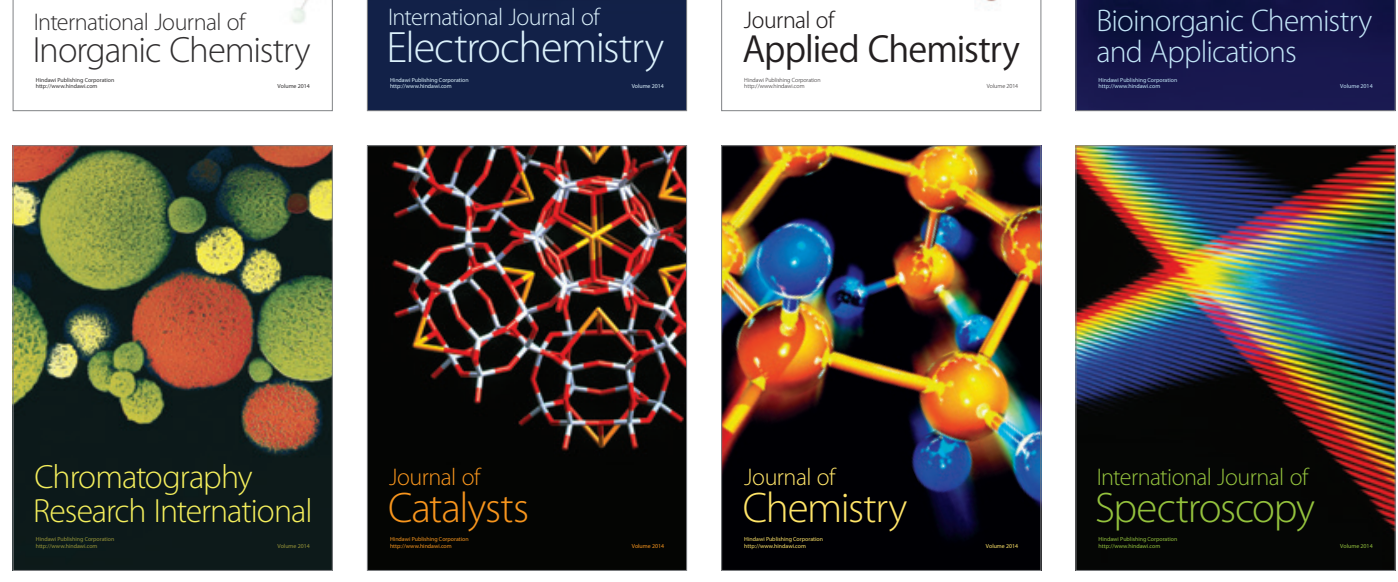